\title{
The momentum kernel of gauge and gravity theories
}

\author{
N.E.J. Bjerrum-Bohr, ${ }^{a}$ Poul H. Damgaard, ${ }^{a}$ Thomas Søndergaard ${ }^{a}$ and \\ Pierre Vanhove ${ }^{b}$ \\ ${ }^{a}$ Niels Bohr International Academy and Discovery Center, The Niels Bohr Institute, \\ Blegdamsvej 17, DK-2100 Copenhagen Ø, Denmark \\ ${ }^{b} C E A, D S M$, Institut de Physique Théorique, IPhT, CNRS, MPPU, \\ URA2306, Saclay, F-91191 Gif-sur-Yvette, France \\ E-mail: bjbohr@nbi.dk, phdamg@nbi.dk, tsonderg@nbi.dk, \\ pierre.vanhove@cea.fr
}

ABSTRACT: We derive an explicit formula for factorizing an $n$-point closed string amplitude into open string amplitudes. Our results are phrased in terms of a momentum kernel which in the limit of infinite string tension reduces to the corresponding field theory kernel. The same momentum kernel encodes the monodromy relations which lead to the minimal basis of color-ordered amplitudes in Yang-Mills theory. There are interesting consequences of the momentum kernel pertaining to soft limits of amplitudes. We also comment on surprising links between gravity and certain combinations of kinematic and color factors in gauge theory.

KEYWords: Supersymmetric gauge theory, Superstrings and Heterotic Strings, Models of Quantum Gravity

ArXiv EPRINT: 1010.3933 


\section{Contents}

1 Introduction 1

2 The momentum kernel in string theory $\quad 3$

2.1 Gravity and gauge theory amplitudes 3

3 Properties of the momentum kernel $\quad 8$

3.1 Minimal basis from momentum kernel 9

4 Soft limit of graviton amplitudes at tree-level $\quad 10$

4.1 A more crossing-symmetric KLT relation from soft limits 11

5 Color and its kinematic factors $\quad 13$

6 Conclusions $\quad 15$

\section{Introduction}

Close to a century after its first formulation, the general theory of relativity continues to give us surprises. In particular, remarkable new insight arises from the intimate relation between gravity and Yang-Mills theories. In perturbation theory one of the most intriguing manifestations of this comes from the factorization of closed string amplitudes into products of open string amplitudes, the Kawai-Lewellen-Tye (KLT) relations [1]. In the field theory limit, these relations show that tree-level amplitudes of gravitons can be expressed as products of color-ordered Yang-Mills amplitudes. Recently, these KLT-relations between gravity amplitudes and Yang-Mills amplitudes have been proven in a series of different but equivalent forms using only concepts from relativistic quantum field theory $[2,3]$. The explicit $n$-point formula that was conjectured in ref. [4] has been proven as one particular case [3].

One interesting by-product of the field theory derivation provided in refs. [2, 3] was the observation that KLT-relations are closely linked to a set of newly discovered identities among gauge theory amplitudes, the BCJ-relations [5]. These BCJ-relations, which also hold when including matter $[6,7]$, turn out to be the field theory limit of monodromy relations in string theory [8] (see also $[9,10]$ ). The field theory proof of KLT-relations uses the same on-shell recursion techniques as the field theory proof of BCJ-relations [11]. But the connection between the two sets of relations runs deeper. Indeed, monodromy relations and hence BCJ-relations are implicitly implied by the analysis of the original KLT paper [1].

Although the recipe for deriving the $n$-point KLT-relations between closed and open string amplitudes was given in ref. [1], a general explicit expression valid for all $n$ has never 
been provided. In this paper we will derive the explicit formulae for any $n$. A central object that emerges is a momentum kernel

$$
\mathcal{S}_{\alpha^{\prime}}\left[i_{1}, \ldots, i_{k} \mid j_{1}, \ldots, j_{k}\right]_{p} \equiv\left(\pi \alpha^{\prime} / 2\right)^{-k} \prod_{t=1}^{k} \sin \left(\pi \alpha^{\prime}\left(p \cdot k_{i_{t}}+\sum_{q>t}^{k} \theta\left(i_{t}, i_{q}\right) k_{i_{t}} \cdot k_{i_{q}}\right)\right),
$$

whose precise definition will be provided below. This momentum kernel maps products of open string amplitudes to closed string amplitudes. In the field theory limit $\alpha^{\prime} \rightarrow 0$ it turns into the field theory momentum kernel $\mathcal{S}$ that maps field theory Yang-Mills amplitudes to gravity amplitudes $[2,3]$. In beautiful agreement with the corresponding observation in field theory, the string theory momentum kernel $\mathcal{S}_{\alpha^{\prime}}$ is precisely the generator of monodromy relations. Phrased more precisely, it annihilates color-ordered amplitudes $\mathcal{A}_{n}$ according to

$$
\sum_{\sigma} \mathcal{S}_{\alpha^{\prime}}[\sigma(2, \ldots, n-1) \mid \beta(2, \ldots, n-1)]_{k_{1}} \mathcal{A}_{n}(n, \sigma(2, \ldots, n-1), 1)=0,
$$

where $\beta$ is any permutation of leg $2, \ldots, n-1$ and the sum runs over all permutations of these legs.

Particularly interesting insight arises if one uses a construction based on the heterotic string [1]. This shows that Yang-Mills amplitudes $A_{n}^{\mathrm{YM}}$ can be expressed as a sum of products of color-ordered Yang-Mills amplitudes $A_{n}, A_{n}^{\mathrm{YM}} \sim \sum A_{n} \mathcal{S}_{\alpha^{\prime}} \widetilde{A}_{n}^{s}$, where $\widetilde{A}_{n}^{s}$ is a scalar amplitude based on vertices that are trivial except for the structure constants of the gauge group [12]. The similarity with the relation for gravity amplitudes $M_{n} \sim \sum A_{n} \mathcal{S}_{\alpha^{\prime}} \widetilde{A}_{n}$ in terms of gauge amplitudes is striking. This leads naturally to an alternative viewpoint on KLT-relations that comes from exploring Jacobi-like identities [5] among numerator factors on the Yang-Mills side. Also this picture, and its generalization to extended Jacobi-like structures, has a natural string theory interpretation [13-15]. This becomes particularly transparent within the framework of the heterotic string [14] (see also [16, 17]). There is hope that much of this tree-level structure $[18,19]$ carries over to any number of loops [20]. This can be used to analyze the ultraviolet behaviour of $\mathcal{N}=8$ supergravity [21]. All of this is strong motivation for analyzing the numerator approach and the associated duality between kinematic and color structures in greater detail.

Finally, an interesting case arises when one applies the field theory momentum kernel $\mathcal{S}$ to amplitudes with mismatched external helicity legs. Then one obtains new non-linear relations among Yang-Mills amplitudes [22] that can be understood in the context of $R$ charges [23-26].

This paper will be devoted to a detailed study of the string theory momentum kernel $\mathcal{S}_{\alpha^{\prime}}$. First we derive, for the first time, the explicit $n$-point relations between closed string and open string amplitudes, and thus demonstrate in detail how the momentum kernel arises in string theory. We next explore some of the properties of this momentum kernel, and in particular we explain how it acts as a generator of monodromy relations. It turns out that there is, just as in field theory [22], a great amount of freedom in writing down the explicit KLT-map, a freedom which is directly related to this monodromy. Common to all rewritings is, however, the same momentum kernel $\mathcal{S}_{\alpha^{\prime}}$. In the last part of the paper we make some observations regarding the momentum kernel $\mathcal{S}$ in the field theory limit 
and soft factorization of amplitudes in gauge theory and gravity. We also comment on the relation to the approach based on Jacobi-like structures among amplitudes.

\section{The momentum kernel in string theory}

In this section we will derive a general form of $n$-point closed string amplitudes in terms of products of color-ordered open string amplitudes. Like in the KLT paper [1], we proceed by explicit holomorphic factorization of a closed string amplitude.

The heterotic and open strings have different spectra that leads to different effective actions. In the field theory $\alpha^{\prime} \rightarrow 0$ limit the heterotic and open string tree-level amplitudes reduce to various gauge or gravity amplitudes. Closed type II and heterotic string tree-level gravity amplitudes are different because of the different spectra in the two theories. $\mathcal{N}=8$ supergravity amplitudes are computed as the field theory limit of closed string type II amplitudes, and $\mathcal{N}=4$ supergravity amplitudes as the field theory limit of heterotic string amplitudes. For multi-graviton amplitudes the difference arises at higher order in $\alpha^{\prime}$ and it does not affect the field theory limit.

When performing the holomorphic factorization of the amplitudes we will not have to specify the actual detailed form of the left and right moving string amplitudes. But we will pay attention to the monodromy properties of the amplitude when the positions of the external states move on the sphere. All the monodromy properties [8, 15] arise from the contraction between the corresponding plane-wave factors. In this way universal relations emerge.

\subsection{Gravity and gauge theory amplitudes}

After fixing the three points $z_{1}=0, z_{n-1}=1$ and $z_{n}=\infty$, the $n$-point closed string amplitude takes the general form

$$
\mathcal{M}_{n}=\left(\frac{i}{2 \pi \alpha^{\prime}}\right)^{n-3} \int_{i=2}^{n-2} d^{2} z_{i}\left|z_{i}\right|^{2 \alpha^{\prime} k_{1} \cdot k_{i}}\left|z_{i}-1\right|^{2 \alpha^{\prime} k_{n-1} \cdot k_{i}} \prod_{i<j \leq n-2}\left|z_{j}-z_{i}\right|^{2 \alpha^{\prime} k_{i} \cdot k_{j}} f\left(z_{i}\right) g\left(\bar{z}_{i}\right),
$$

where $f\left(z_{i}\right)$ and $g\left(\bar{z}_{i}\right)$ arise from the operator product expansion of the vertex operators. They are functions without branch cuts. The precise form of these functions depends on the external states. They can be gravitons or gauge fields, or, in the straightforward supersymmetric generalization, any part of the $\mathcal{N}=4$ supermultiplet. However, the exact form of these functions will not affect the general relations. For definiteness, let us from now on consider $\mathcal{M}_{n}$ to be a gravity amplitude.

Following $[27,28]$ we can write eq. (2.1) in terms of $v_{i}^{1}$ and $v_{i}^{2}$, where $z_{i}=v_{i}^{1}+i v_{i}^{2}$, and then make the following change of variables for $v_{i}^{2}$

$$
v_{i}^{2} \longrightarrow i e^{-2 i \epsilon} v_{i}^{2}
$$

where $\epsilon>0$ is some small number. Keeping only the terms linear in $\epsilon$, i.e. using

$$
i e^{-2 i \epsilon} v_{i}^{2} \simeq i(1-2 i \epsilon) v_{i}^{2}
$$


and introducing the notation

$$
v_{i}^{ \pm} \equiv v_{i}^{1} \pm v_{i}^{2}, \quad \delta_{i} \equiv v_{i}^{+}-v_{i}^{-},
$$

we can write eq. (2.1) as an 'almost factorized' amplitude

$$
\begin{aligned}
\mathcal{M}_{n}= & \left(\frac{i}{2}\right)^{n-3}\left(\frac{i}{2 \pi \alpha^{\prime}}\right)^{n-3} \int_{-\infty}^{+\infty} \prod_{i=2}^{n-2} d v_{i}^{+} d v_{i}^{-} f\left(v_{i}^{-}\right) g\left(v_{i}^{+}\right) \\
& \times\left(v_{i}^{+}-i \epsilon \delta_{i}\right)^{\alpha^{\prime} k_{1} \cdot k_{i}}\left(v_{i}^{-}+i \epsilon \delta_{i}\right)^{\alpha^{\prime} k_{1} \cdot k_{i}}\left(v_{i}^{+}-1-i \epsilon \delta_{i}\right)^{\alpha^{\prime} k_{n-1} \cdot k_{i}}\left(v_{i}^{-}-1+i \epsilon \delta_{i}\right)^{\alpha^{\prime} k_{n-1} \cdot k_{i}} \\
& \times \prod_{i<j \leq n-2}\left(v_{i}^{+}-v_{j}^{+}-i \epsilon\left(\delta_{i}-\delta_{j}\right)\right)^{\alpha^{\prime} k_{i} \cdot k_{j}}\left(v_{i}^{-}-v_{j}^{-}+i \epsilon\left(\delta_{i}-\delta_{j}\right)\right)^{\alpha^{\prime} k_{i} \cdot k_{j}}
\end{aligned}
$$

The appearance of branch cuts in the integrand is crucial. We have the following choices for $x^{\alpha}$ when $x<0$

$$
x^{\alpha}= \begin{cases}e^{i \pi \alpha}(-x)^{\alpha} & \operatorname{Im}(x) \geq 0, \\ e^{-i \pi \alpha}(-x)^{\alpha} & \operatorname{Im}(x)<0 .\end{cases}
$$

By splitting the $v_{i}^{+}$-integrals and using symmetry, the $n$-point graviton amplitude can be written as

$$
\mathcal{M}_{n}=\sum_{\sigma} M_{n}(\sigma(2), \ldots, \sigma(n-2)),
$$

where $M_{n}(\sigma(2), \ldots, \sigma(n-2))$ is the ordered amplitude defined such that $v_{\sigma(2)}^{+}<v_{\sigma(3)}^{+}<$ $\cdots<v_{\sigma(n-2)}^{+}$.

If one of the $v_{i}^{+}$is in the interval ] $-\infty, 0\left[\right.$ the integral contours for $v_{i}^{-}$lie below the real axis and the integral vanishes. This is because there are no poles of the functions $f$ and $g$ outside the real axis, and the integrand nicely vanishes at infinity. By the same reasoning, if one of the $v_{i}^{+}$is in the interval $] 1,+\infty\left[\right.$, the integral contours for $v_{i}^{-}$lie above the real axis and the integral is again vanishing.

Therefore, to get a non-vanishing contribution, all the $v_{+}$'s need to be distributed in the interval $] 0,1\left[: 0<v_{\sigma(2)}^{+}<v_{\sigma(3)}^{+}<\cdots<v_{\sigma(n-2)}^{+}<1\right.$ where $\sigma$ denotes a permutation of the $(n-3)$ ! labels. These $v_{i}^{+}$integrals lead to

$$
\begin{aligned}
& \mathcal{A}_{n}(1, \sigma(2, \ldots, n-2), n-1, n)=\int_{0<v_{\sigma(2)}^{+}<\cdots<v_{\sigma(n-2)}^{+}<1} \prod_{i=2}^{n-2} d v_{\sigma(i)}^{+} g\left(v_{\sigma(i)}^{+}\right) \\
& \times\left(v_{\sigma(i)}^{+}\right)^{\alpha^{\prime} k_{1} \cdot k_{\sigma(i)}}\left(1-v_{\sigma(i)}^{+}\right)^{\alpha^{\prime} k_{n-1} \cdot k_{\sigma(i)}} \prod_{\sigma(i)<\sigma(j) \leq n-2}\left(v_{\sigma(j)}^{+}-v_{\sigma(i)}^{+}\right)^{\alpha^{\prime} k_{\sigma(i)} \cdot k_{\sigma(j)}}
\end{aligned}
$$

which are integrals corresponding to color-ordered open string amplitudes.

We now turn to the evaluation of the integrals over the $v_{i}^{-}$variables. This will lead to ordered integrals $\widetilde{\mathcal{A}}_{n}$ that correspond to the 'right-moving' sectors. For simplicity we just consider the case of ordering $\{2,3, \ldots, n-2\}$ of the $v_{i}^{+}$variables. All other cases are obtained by summing over the permutations of these $n-3$ variables. 


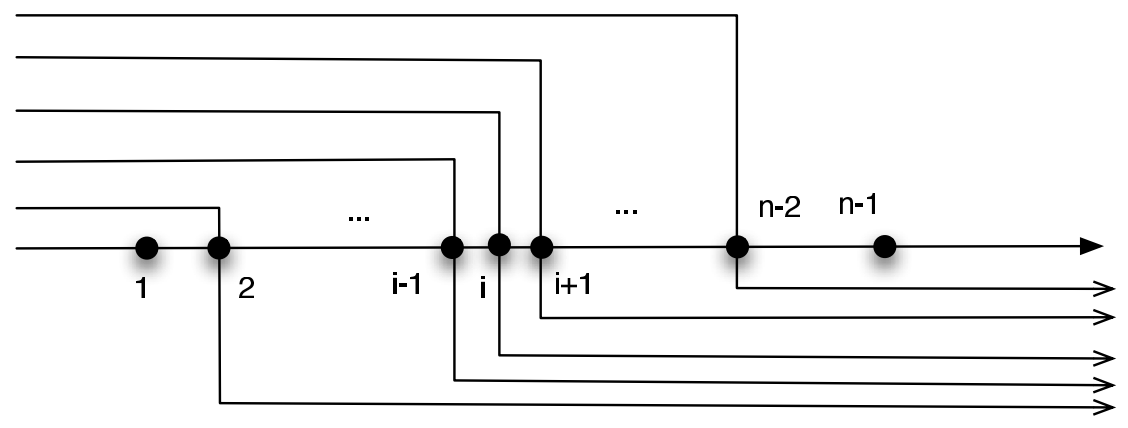

Figure 1. The nested structure of the contours of integration for the variable $v_{i}^{-}$corresponding to the ordering $0<v_{2}^{+}<v_{3}^{+}<\cdots<v_{n-2}^{+}<1$ of the $v_{+}$variables.

For each $2 \leq i \leq n-2$ we first examine the behavior of the integrand of (2.1) around the different branch cuts. For $v_{i}^{-} \sim 0$ the quantity $v_{i}^{-}+i \epsilon \delta_{i} \sim v_{i}^{-}+i \epsilon v_{i}^{+}$has a positive imaginary part. Therefore the contour is above the real axis. For $v_{i}^{-} \sim 1$ the quantity $v_{i}^{-}-1+i \epsilon \delta_{i} \sim v_{i}^{-}-1+i \epsilon\left(v_{i}^{+}-1\right)$ has a negative imaginary part; the contour of integration lies below the real axis. Finally, for $v_{i}^{-} \sim v_{j}^{-}$with $i<j$, the quantity $v_{i}^{-}-v_{j}^{-}+i \epsilon\left(\delta_{i}-\delta_{j}\right) \sim v_{i}^{-}-v_{j}^{-}+i \epsilon\left(v_{i}^{+}-v_{j}^{+}\right)$has a negative imaginary part. Therefore the contour of integration for $v_{i}^{-}$goes below the contour of $v_{j}^{-}$for $i<j$. We have represented this nested structure of the contours of integration for the $v_{i}^{-}$variables in figure 1 .

We now consider the deformations of the contours of integration for the $v_{i}^{-}$variables. Because the contours cannot cross each other we need to close them either to the right, turning around the branch cut at $z=1$ by starting with the rightmost, or close the contours to the left, turning around the branch cut at $z=0$, starting with the leftmost.

There is evidently an arbitrariness in the number of contours that are closed to the left or closed to the right. For a given $2 \leq j \leq n-2$, we can pull the contours for the set between 2 and $j-1$ to the left, and the set between $j$ and $n-2$ to the right.

Pulling the contour for 2 gives

$$
\begin{aligned}
& \int_{C_{2}} d v_{2}^{-}\left(v_{2}^{-}\right)^{\alpha^{\prime} k_{1} \cdot k_{2}}\left(1-v_{2}^{-}\right)^{\alpha^{\prime} k_{n-1} \cdot k_{2}} \prod_{j=3}^{n-2}\left(v_{j}^{-}-v_{2}^{-}\right)^{\alpha^{\prime} k_{j} \cdot k_{2}} f\left(v_{2}^{-}\right) \\
& =2 i \sin \left(\pi \alpha^{\prime} k_{1} \cdot k_{2}\right) \int_{-\infty}^{0} d v_{2}^{-}\left(-v_{2}^{-}\right)^{\alpha^{\prime} k_{1} \cdot k_{2}}\left(1-v_{2}^{-}\right)^{\alpha^{\prime} k_{n-1} \cdot k_{2}} \prod_{j=3}^{n-2}\left(v_{j}^{-}-v_{2}^{-}\right)^{\alpha^{\prime} k_{j} \cdot k_{2}} f\left(v_{2}^{-}\right) .
\end{aligned}
$$

Here we have explicitly shown only the contributions where $v_{2}^{-}$has branch cuts.

Closing the contour for $v_{3}^{-}$to the left leads to

$$
\begin{aligned}
& \int_{C_{3}} d v_{3}^{-}\left(v_{3}^{-}\right)^{\alpha^{\prime} k_{1} \cdot k_{3}}\left(1-v_{3}^{-}\right)^{\alpha^{\prime} k_{n-1} \cdot k_{3}}\left(v_{3}^{-}-v_{2}^{-}\right)^{\alpha^{\prime} k_{2} \cdot k_{3}} \prod_{j=4}^{n-2}\left(v_{j}^{-}-v_{3}^{-}\right)^{\alpha^{\prime} k_{j} \cdot k_{3}} f\left(v_{3}^{-}\right) \\
& =2 i \sin \left(\pi \alpha^{\prime} k_{1} \cdot k_{3}\right) \int_{v_{2}^{-}}^{0} d v_{3}^{-}\left(-v_{3}^{-}\right)^{\alpha^{\prime} k_{1} \cdot k_{3}}\left(1-v_{3}^{-}\right)^{\alpha^{\prime} k_{n-1} \cdot k_{3}}\left(v_{3}^{-}-v_{2}^{-}\right)^{\alpha^{\prime} k_{2} \cdot k_{3}}
\end{aligned}
$$




$$
\begin{aligned}
& \times \prod_{j=4}^{n-2}\left(v_{j}^{-}-v_{3}^{-}\right)^{\alpha^{\prime} k_{j} \cdot k_{3}} f\left(v_{3}^{-}\right) \\
+2 i \sin \left(\pi \alpha^{\prime}\left(k_{1}+k_{2}\right) \cdot k_{3}\right) \int_{-\infty}^{v_{2}^{-}} d v_{3}^{-}\left(-v_{3}^{-}\right)^{\alpha^{\prime} k_{1} \cdot k_{3}}\left(1-v_{3}^{-}\right)^{\alpha^{\prime} k_{n-1} \cdot k_{3}}\left(v_{2}^{-}-v_{3}^{-}\right)^{\alpha^{\prime} k_{2} \cdot k_{3}} & \\
& \times \prod_{j=4}^{n-2}\left(v_{j}^{-}-v_{3}^{-}\right)^{\alpha^{\prime} k_{j} \cdot k_{3}} f\left(v_{3}^{-}\right),
\end{aligned}
$$

and so on until one has pulled the contour for $v_{j-1}^{-}$to the left.

When closing the contours to the right we start from the contour for $v_{n-2}^{-}$down to the one for $v_{j}^{-}$. Pulling first the contour for $v_{n-2}^{-}$to the right leads to

$$
\begin{gathered}
\int_{C_{n-2}} d v_{n-2}^{-}\left(v_{n-2}^{-}\right)^{\alpha^{\prime} k_{1} \cdot k_{n-2}\left(1-v_{n-2}^{-}\right)^{\alpha^{\prime} k_{n-1} \cdot k_{n-2}}} \prod_{j=2}^{n-3}\left(v_{n-2}^{-}-v_{j}^{-}\right)^{\alpha^{\prime} k_{j} \cdot k_{n-2}} f\left(v_{n-2}^{-}\right) \\
=2 i \sin \left(\pi \alpha^{\prime} k_{n-1} \cdot k_{n-2}\right) \int_{1}^{+\infty} d v_{n-2}^{-}\left(v_{n-2}^{-}\right)^{\alpha^{\prime} k_{1} \cdot k_{n-2}}\left(v_{n-2}^{-}-1\right)^{\alpha^{\prime} k_{n-1} \cdot k_{n-2}} \\
\times \prod_{j=2}^{n-3}\left(v_{n-2}^{-}-v_{j}^{-}\right)^{\alpha^{\prime} k_{j} \cdot k_{n-2}} f\left(v_{n-2}^{-}\right) .
\end{gathered}
$$

Similarly, closing the contour for $v_{n-3}^{-}$to the right leads to

$$
\begin{aligned}
& \int_{C_{n-3}} d v_{n-3}^{-}\left(v_{3}^{-}\right)^{\alpha^{\prime} k_{1} \cdot k_{n-3}}\left(1-v_{n-3}^{-}\right)^{\alpha^{\prime} k_{n-1} \cdot k_{n-3}}\left(v_{n-2}^{-}-v_{n-3}^{-}\right)^{\alpha^{\prime} k_{n-2} \cdot k_{n-3}} \\
& \times \prod_{j=2}^{n-4}\left(v_{n-3}^{-}-v_{j}^{-}\right)^{\alpha^{\prime} k_{j} \cdot k_{n-3}} f\left(v_{n-3}^{-}\right) \\
& =2 i \sin \left(\pi \alpha^{\prime} k_{n-1} \cdot k_{n-3}\right) \int_{1}^{v_{n-2}^{-}} d v_{n-3}^{-}\left(v_{n-3}^{-}\right)^{\alpha^{\prime} k_{1} \cdot k_{n-3}}\left(v_{n-3}^{-}-1\right)^{\alpha^{\prime} k_{n-1} \cdot k_{n-3}} \\
& \times\left(v_{n-2}^{-}-v_{n-3}^{-}\right)^{\alpha^{\prime} k_{n-2} \cdot k_{n-3}} \prod_{j=2}^{n-4}\left(v_{n-3}^{-}-v_{j}^{-}\right)^{\alpha^{\prime} k_{j} \cdot k_{n-3}} f\left(v_{n-3}^{-}\right) \\
& +2 i \sin \left(\pi \alpha^{\prime}\left(k_{n-1}+k_{n-2}\right) \cdot k_{n-3}\right) \int_{v_{n-2}^{-}}^{+\infty} d v_{n-3}^{-}\left(v_{n-3}^{-}\right)^{\alpha^{\prime} k_{n-1} \cdot k_{n-3}}\left(v_{n-3}^{-}-1\right)^{\alpha^{\prime} k_{n-1} \cdot k_{n-3}} \\
& \times\left(v_{n-3}^{-}-v_{n-2}^{-}\right)^{\alpha^{\prime} k_{n-2} \cdot k_{n-3}} \prod_{j=2}^{n-4}\left(v_{n-3}^{-}-v_{j}^{-}\right)^{\alpha^{\prime} k_{j} \cdot k_{n-3}} f\left(v_{n-3}^{-}\right),
\end{aligned}
$$

and so on until one reaches the contour for $v_{j}^{-}$. The integrals over the $v^{-}$variables are the ordered string amplitudes $\widetilde{\mathcal{A}}_{n}(\gamma(2, \ldots, j-1), 1, n-1, \beta(j, \ldots, n-2), n)$.

Collecting these contour deformations lead to the following expression for the $v^{-}$part of the integral in (2.1)

$$
\begin{aligned}
(-i / 4)^{n-3} \sum_{\gamma} \sum_{\beta} \mathcal{S}_{\alpha^{\prime}}[\gamma(2, \ldots, j-1) \mid 2, \ldots, j-1]_{k_{1}} \mathcal{S}_{\alpha^{\prime}}[\beta(j, \ldots, n-2) \mid j, \ldots, n-2]_{k_{n-1}} \\
\times \widetilde{\mathcal{A}}_{n}(\gamma(2, \ldots, j-1), 1, n-1, \beta(j, \ldots, n-2), n)
\end{aligned}
$$


which is of course multiplied by the left-moving amplitude $\mathcal{A}_{n}(1,2, \ldots, n)$ from the integral over the $v^{+}$variables. Here we also see the first appearance of the momentum kernel $\mathcal{S}_{\alpha^{\prime}}[\gamma, \sigma]_{p}$. It depends on the permutations $\gamma$ of the $v_{i}^{-}$-variables and the ordering of the $v_{i}^{+}$. It also depends on the momenta $p=k_{1}$ and $k_{n-1}$ of the states at the branch cut at $z=0$ or $z=1$ on the sphere. In general it can be defined as

$$
\mathcal{S}_{\alpha^{\prime}}\left[i_{1}, \ldots, i_{k} \mid j_{1}, \ldots, j_{k}\right]_{p} \equiv\left(\pi \alpha^{\prime} / 2\right)^{-k} \prod_{t=1}^{k} \sin \left(\pi \alpha^{\prime}\left(p \cdot k_{i_{t}}+\sum_{q>t}^{k} \theta\left(i_{t}, i_{q}\right) k_{i_{t}} \cdot k_{i_{q}}\right)\right)
$$

where $\theta\left(i_{t}, i_{q}\right)$ equals 1 if the ordering of the legs $i_{t}$ and $i_{q}$ is opposite in the sets $\left\{i_{1}, \ldots, i_{k}\right\}$ and $\left\{j_{1}, \ldots, j_{k}\right\}$, and 0 if the ordering is the same.

We have normalized this expression so that in the field theory limit $\alpha^{\prime} \rightarrow 0$ the kernel $\mathcal{S}_{\alpha^{\prime}}$ reduces to the field theory kernel $\mathcal{S}$ of $[2,3,22]$. As indicated, we will distinguish between these two momentum kernels by putting a subscript $\alpha^{\prime}$ on the one of string theory. In the next section we will analyze the properties of this kernel.

To get the full closed string amplitude (2.1) we need to multiply the left-moving amplitude of the $v^{+}$integrations in (2.8) with the right-moving contribution in (2.13) and then sum over all orderings to get

$$
\begin{aligned}
& \mathcal{M}_{n}=(-i / 4)^{n-3} \times \\
& \sum_{\sigma} \sum_{\gamma, \beta} \mathcal{S}_{\alpha^{\prime}}[\gamma(\sigma(2), \ldots, \sigma(j-1)) \mid \sigma(2, \ldots, j-1)]_{k_{1}} \mathcal{S}_{\alpha^{\prime}}[\beta(\sigma(j), \ldots, \sigma(n-2)) \mid \sigma(j, \ldots, n-2)]_{k_{n-1}} \\
& \quad \times \mathcal{A}_{n}(1, \sigma(2, \ldots, n-2), n-1, n) \widetilde{\mathcal{A}}_{n}(\gamma(\sigma(2), \ldots, \sigma(j-1)), 1, n-1, \beta(\sigma(j), \ldots, \sigma(n-2)), n) .
\end{aligned}
$$

This provide a general form of the closed/open string relation between external gauge bosons and gravitons at tree-level. When restricted to the case of graviton external states the field theory limit of this expression reduces to the form derived in [3, 22].

As seen from the above derivation, expression (2.15) is actually independent of the value of $j$. This $j$-independence reflects the arbitrariness in the number of contours one closes to the left around the branch point at $z=0$ or to the right around the branch point at $z=1$. As further explained below, independence under shifts of $j$ is a consequence of the monodromy relations [8] that are satisfied by the color-ordered string amplitudes.

The expression $(2.15)$ is a sum over $(n-3) ! \times(j-2) ! \times(n-1-j) !$ terms. The number of terms takes the maximal value $(n-3) ! \times(n-3)$ ! for $j=2$ or $j=n-1$. The choice made by KLT in [1] consists in closing half of the contours to the left and and the other half to the right, i.e. $j=\lceil n / 2\rceil$. This leads to the smallest number of terms $(n-3) ! \times\left(\left\lceil\frac{n}{2}\right\rceil-2\right) ! \times\left(\left\lfloor\frac{n}{2}\right\rfloor-\right.$ 1)! (The floor and ceiling functions are defined on half-integers as follows: $\lfloor n / 2\rfloor=(n-1) / 2$ if $n$ is odd, or $n / 2$ if $n$ is even. $\lceil n / 2\rceil=(n+1) / 2$ if $n$ is odd, or $n / 2$ if $n$ is even.) For $j=n-1$ the amplitude takes the nice form

$$
\begin{aligned}
\mathcal{M}_{n}=(-1)^{n-3} \sum_{\sigma, \gamma} \mathcal{S}_{\alpha^{\prime}}[\gamma(2, \ldots, n-2) \mid \sigma(2, \ldots, n-2)]_{k_{1}} \\
\quad \times \mathcal{A}_{n}(1, \sigma(2, \ldots, n-2), n-1, n) \widetilde{\mathcal{A}}_{n}(n-1, n, \gamma(2, \ldots, n-2), 1) .
\end{aligned}
$$




\section{Properties of the momentum kernel}

The $\mathcal{S}_{\alpha^{\prime}}$ kernel has a number of fundamental properties that correspond to those of the field theory kernel $\mathcal{S}$. In field theory these properties ensure, for instance, the correct cancellation of poles between products of color-ordered gauge amplitudes and correct factorization properties compatible with on-shell recursion techniques. The $\mathcal{S}_{\alpha^{\prime}}$ version of these properties can be seen to hold by the same kind of arguments used for the field theory $\mathcal{S}$ kernel in $[2,3,22]$, and will therefore not be repeated here.

(1) Reflection symmetry:

$$
\mathcal{S}_{\alpha^{\prime}}[\sigma(1, \ldots, k) \mid \gamma(1, \ldots, k)]_{p}=\mathcal{S}_{\alpha^{\prime}}[\gamma(k, \ldots, 1) \mid \sigma(k, \ldots, 1)]_{p},
$$

where $p$ is a massless momentum and $\sigma$ and $\gamma$ are arbitrary permutations of the $k$ labels $\{1, \ldots, k\}$.

(2) Factorization:

Assuming that $P \equiv k_{1}+k_{2}+\cdots+k_{p}$ is on-shell, i.e. $P^{2}=0$, the following factorization holds

$$
\begin{aligned}
& \mathcal{S}_{\alpha^{\prime}}[\gamma(p+1, \ldots, k) \sigma(2, \ldots, p) \mid \beta(2, \ldots, p) \delta(p+1, \ldots, k)]_{k_{1}} \\
& =\mathcal{S}_{\alpha^{\prime}}[\sigma(2, \ldots, p) \mid \beta(2, \ldots, p)]_{k_{1}} \times \mathcal{S}_{\alpha^{\prime}}[\gamma(p+1, \ldots, k) \mid \delta(p+1, \ldots, k)]_{P},
\end{aligned}
$$

where $\alpha, \beta, \gamma$ and $\delta$ are arbitrary permutations.

(3) Annihilation of amplitudes:

$$
\sum_{\sigma} \mathcal{S}_{\alpha^{\prime}}[\sigma(2, \ldots, n-1) \mid \beta(2, \ldots, n-1)]_{k_{1}} \mathcal{A}_{n}(n, \sigma(2, \ldots, n-1), 1)=0,
$$

where $\beta$ is any permutation of the legs $\{2, \ldots, n-1\}$ and $\mathcal{A}_{n}$ are color-ordered treelevel string amplitudes.

(4) The shifting-formula for $j$ :

By using that formula (2.15) is independent of $j$ we obtain the following relation, which is valid for any $2 \leq j \leq n-2$ :

$$
\begin{gathered}
\sum_{\gamma, \beta} \mathcal{S}_{\alpha^{\prime}}\left[\gamma\left(i_{2}, \ldots, i_{j}\right) \mid i_{2}, \ldots, i_{j}\right]_{k_{1}} \mathcal{S}_{\alpha^{\prime}}\left[i_{j+1}, \ldots, i_{n-2} \mid \beta\left(i_{j+1}, \ldots, i_{n-2}\right)\right]_{k_{n-1}} \\
\times \mathcal{A}_{n}\left(\gamma\left(i_{2}, \ldots, i_{j}\right), 1, n-1, \beta\left(i_{j+1}, \ldots, i_{n-2}\right), n\right) \\
=\sum_{\gamma^{\prime}, \beta^{\prime}} \mathcal{S}_{\alpha^{\prime}}\left[\gamma^{\prime}\left(i_{2}, \ldots, i_{j-1}\right) \mid i_{2}, \ldots, i_{j-1}\right]_{k_{1}} \mathcal{S}_{\alpha^{\prime}}\left[i_{j}, \ldots, i_{n-2} \mid \beta^{\prime}\left(i_{j}, \ldots, i_{n-2}\right)\right]_{k_{n-1}} \\
\times \mathcal{A}_{n}\left(\gamma^{\prime}\left(i_{2}, \ldots, i_{j-1}\right), 1, n-1, \beta^{\prime}\left(i_{j}, \ldots, i_{n-2}\right), n\right)
\end{gathered}
$$

They are particular cases of linear monodromy relations satisfied by the color-ordered amplitudes. Conversely, monodromy relations are necessary and sufficient for proving the $j$-independence of the general KLT formula (2.15). Such monodromy properties are generated by the contour deformations as discussed in section 2.1. 
As mentioned, property (1)-(4) are also satisfied in field theory simply by replacing $\mathcal{S}_{\alpha^{\prime}}$ by its field theory limit $\mathcal{S}$ and replacing the color-ordered string amplitudes $\mathcal{A}_{n}$ by their corresponding field theory limits $A_{n}$.

\subsection{Minimal basis from momentum kernel}

One can write the equations in $(3.3)$ as a linear system of the $(n-2) !-(n-3) !=$ $(n-3) \times(n-3)$ ! amplitudes $\mathcal{A}_{n}(n, \sigma(2), \ldots, \sigma(i-1), n-1, \sigma(i), \ldots, \sigma(n-2), 1)$ with a right-hand-side expressed only in a minimal basis of size $(n-3)$ !, spanned by the amplitudes $\mathcal{A}_{n}(n, \sigma(2), \ldots, \sigma(n-2), n-1,1)$

$\sum_{i=2}^{n-2} \sum_{\sigma} \mathcal{S}_{\alpha^{\prime}}[\sigma(2, \ldots, n-1) \mid \beta(2, \ldots, n-1)]_{k_{1}} \mathcal{A}_{n}(n, \sigma(2), \ldots, \sigma(i-1), n-1, \sigma(i), \ldots, \sigma(n-2), 1)$
$=-\sum_{\sigma} \mathcal{S}_{\alpha^{\prime}}[\sigma(2, \ldots, n-2), n-1 \mid \beta(2, \ldots, n-1)]_{k_{1}} \mathcal{A}_{n}(n, \sigma(2), \ldots, \sigma(n-2), n-1,1)$.

The resulting linear system is non-degenerate for a generic choice of momenta and can be inverted to express the amplitudes $\mathcal{A}_{n}(n, \sigma(2), \ldots, \sigma(i-1), n-1, \sigma(i), \ldots, \sigma(n-2), 1)$ in the basis of $\mathcal{A}_{n}(n, \sigma(2), \ldots, \sigma(n-2), n-1,1)$. Equivalently, the rank of the square matrix of size $(n-2)$ !, with entries $S[\sigma \mid \beta]$, has a kernel of dimension $(n-3)$ ! and the linear system for the amplitudes $\mathcal{A}_{n}(n, \sigma(2), \ldots, \sigma(i-1), n-1, \sigma(i), \ldots, \sigma(n-2), 1)$ in $(3.5)$ is $(n-3) \times(n-3)$ !. We have numerically check this fact up to 10 points.

Below we work out some specific examples. Especially, we will illustrate how the monodromy relations with leg $n$ and 1 fixed as in (3.3) are enough [11] to deduce the minimal basis [8].

At four points the momentum kernel takes the form (discarding the overall normalization constant)

$$
\begin{aligned}
& \mathcal{S}_{\alpha^{\prime}}[23 \mid 23]_{k_{1}}=\mathcal{S}_{\alpha^{\prime}}[32 \mid 32]_{k_{1}}=\sin \left(\pi \alpha^{\prime} k_{1} \cdot k_{2}\right) \sin \left(\pi \alpha^{\prime} k_{1} \cdot k_{3}\right), \\
& \mathcal{S}_{\alpha^{\prime}}[23 \mid 32]_{k_{1}}=-\sin \left(\pi \alpha^{\prime} k_{1} \cdot k_{3}\right)^{2}, \quad \mathcal{S}_{\alpha^{\prime}}[32 \mid 23]_{k_{1}}=-\sin \left(\pi \alpha^{\prime} k_{1} \cdot k_{2}\right)^{2},
\end{aligned}
$$

and the system of equations (3.5) gives for $\beta(2,3)=\{2,3\}$ and $\beta(2,3)=\{3,2\}$

$$
\begin{aligned}
\sin \left(\pi \alpha^{\prime} k_{1} \cdot k_{2}\right) \sin \left(\pi \alpha^{\prime} k_{1} \cdot k_{3}\right) \mathcal{A}_{4}(4,2,3,1)-\sin \left(\pi \alpha^{\prime} k_{1} \cdot k_{2}\right)^{2} \mathcal{A}_{4}(4,3,2,1) & =0, \\
-\sin \left(\pi \alpha^{\prime} k_{1} \cdot k_{3}\right)^{2} \mathcal{A}_{4}(4,2,3,1)+\sin \left(\pi \alpha^{\prime} k_{1} \cdot k_{2}\right) \sin \left(\pi \alpha^{\prime} k_{1} \cdot k_{3}\right) \mathcal{A}_{4}(4,3,2,1) & =0 .
\end{aligned}
$$

These two equations are identical and lead to the monodromy relation

$$
\sin \left(\pi \alpha^{\prime} k_{1} \cdot k_{3}\right) \mathcal{A}_{4}(4,2,3,1)=\sin \left(\pi \alpha^{\prime} k_{1} \cdot k_{2}\right) \mathcal{A}_{4}(4,3,2,1)
$$

reducing to a minimal basis.

The system of equations (3.5) for $\beta(2,3,4)=\{2,3,4\}$ and $\beta(2,3,4)=\{3,2,4\}$ implies that

$$
\begin{aligned}
& \sin \left(\pi \alpha^{\prime} k_{1} \cdot k_{2}\right) \sin \left(\pi \alpha^{\prime} k_{1} \cdot k_{3}\right) F(4\{23\})+\sin \left(\pi \alpha^{\prime} k_{1} \cdot k_{2}\right) \sin \left(\pi \alpha^{\prime} k_{3} \cdot\left(k_{1}+k_{2}\right)\right) F(4\{32\})=0, \\
& \sin \left(\pi \alpha^{\prime} k_{1} \cdot k_{3}\right) \sin \left(\pi \alpha^{\prime} k_{2} \cdot\left(k_{1}+k_{3}\right)\right) F(4\{23\})+\sin \left(\pi \alpha^{\prime} k_{1} \cdot k_{2}\right) \sin \left(\pi \alpha^{\prime} k_{1} \cdot k_{3}\right) F(4\{32\})=0,
\end{aligned}
$$


where we have defined

$$
\begin{aligned}
F(4\{23\}) \equiv & -\sin \left(\pi \alpha^{\prime} k_{4} \cdot k_{5}\right) \mathcal{A}_{5}(5,4,2,3,1)+\sin \left(\pi \alpha^{\prime} k_{4} \cdot\left(k_{1}+k_{3}\right)\right) \mathcal{A}_{5}(5,2,4,3,1) \\
& +\sin \left(\pi \alpha^{\prime} k_{1} \cdot k_{4}\right) \mathcal{A}_{5}(5,2,3,4,1), \\
F(4\{32\}) \equiv & -\sin \left(\pi \alpha^{\prime} k_{4} \cdot k_{5}\right) \mathcal{A}_{5}(5,4,3,2,1)+\sin \left(\pi \alpha^{\prime} k_{4} \cdot\left(k_{1}+k_{2}\right)\right) \mathcal{A}_{5}(5,3,4,2,1) \\
& +\sin \left(\pi \alpha^{\prime} k_{1} \cdot k_{4}\right) \mathcal{A}_{5}(5,3,2,4,1) .
\end{aligned}
$$

For generic values of the external momenta the determinant of this system $\sin \left(\pi \alpha^{\prime} k_{1}\right.$. $\left.k_{2}\right) \sin \left(\pi \alpha^{\prime} k_{1} \cdot k_{3}\right) \sin \left(\pi \alpha^{\prime} k_{2} \cdot k_{3}\right) \sin \left(\pi \alpha^{\prime} k_{4} \cdot k_{5}\right)$ is non vanishing, implying that $F(4\{23\})=0$ and $F(4\{32\})=0$. These identities are two of the six monodromy relations [8] that one obtains by keeping four legs fixed and rotating the contour of one external leg (here leg 2). We have seen here how these relations arise from the annihilation property (3).

These two relations directly express the color-ordered amplitudes $\mathcal{A}_{5}(5,3,4,2,1)$ and $\mathcal{A}_{5}(5,2,3,4,1)$ in the minimal basis $\mathcal{A}_{5}(1,2,3,4,5)$ and $\mathcal{A}_{5}(1,3,2,4,5)$. By considering all the other permutations of the external legs it is straightforward to see that property (3) implies that all color-ordered amplitudes can be expressed in the minimal basis $\mathcal{A}_{5}(1,2,3,4,5)$ and $\mathcal{A}_{5}(1,3,2,4,5)$. The generalization to higher $n$-point cases follows analogously.

\section{Soft limit of graviton amplitudes at tree-level}

In this and in the following section we make some observations on the field theory limit.

Interestingly, tree-level gravity amplitudes have a universal behavior when taking one graviton to be soft, a classic result due to Weinberg $[4,29]$

$$
\lim _{k_{s}^{ \pm} \rightarrow 0} M_{n}\left(\ldots, a, s^{ \pm}, b, \ldots\right)=S^{\text {gravity }}\left(s^{ \pm}\right) \times M_{n-1}(\ldots, a, b, \ldots) .
$$

For definiteness take leg $n$ to be soft, then the 'soft factor' is given by the sum

$$
S^{\text {gravity }}\left(n^{ \pm}\right)=\sum_{i=1}^{n-1} s_{n i} S^{\mathrm{YM}}\left(q_{L}, n^{ \pm}, i\right) S^{\mathrm{YM}}\left(q_{R}, n^{ \pm}, i\right),
$$

where $S^{\mathrm{YM}}\left(q_{L}, n^{ \pm}, i\right)$ is the corresponding soft factor for Yang-Mills theory

$$
\lim _{k_{s}^{ \pm} \rightarrow 0} A_{n}\left(\cdots, a, s^{ \pm}, b, \ldots\right)=S^{\mathrm{YM}}\left(a, s^{ \pm}, b\right) A_{n-1}(\cdots, a, b, \ldots) .
$$

It depends on the helicity of the soft gluon [30] and is given by

$$
S^{\mathrm{YM}}\left(q, k^{ \pm}, p\right)=\frac{\epsilon^{ \pm}(q, k) \cdot p}{k \cdot p},
$$

where $\epsilon$ is the polarization vector of the gluon with momentum $k$. The covariant expression for the soft factor of the graviton is therefore

$$
S^{\text {gravity }}\left(n^{ \pm}\right)=\sum_{i=1}^{n-1} \frac{\epsilon^{ \pm}\left(q_{L}, k_{n}\right) \cdot k_{i} \epsilon^{ \pm}\left(q_{R}, k_{n}\right) \cdot k_{i}}{k_{n} \cdot k_{i}},
$$


where the graviton polarization tensor $\epsilon^{ \pm \pm}$has been split into a product of Yang-Mills polarizations $\epsilon^{ \pm} \otimes \epsilon^{ \pm}$. This expression shows explicitly that the gravity soft factor in (4.2) is independent of the choice of reference momenta $q_{L}$ and $q_{R}$. Using

$$
\epsilon^{ \pm}\left(q_{L}, k_{n}\right) \cdot k_{i}-\epsilon^{ \pm}\left(\tilde{q}_{L}, k_{n}\right) \cdot k_{i}=S^{\mathrm{YM}}\left(q_{L}, k_{n}^{ \pm}, \tilde{q}_{L}\right) k_{n} \cdot k_{i},
$$

the soft factor of the graviton changes by

$$
\delta S^{\text {gravity }}\left(n^{ \pm}\right)=S^{\mathrm{YM}}\left(q_{L}, k_{n}^{ \pm}, \tilde{q}_{L}\right) \sum_{i=1}^{n-1} \epsilon^{ \pm}\left(q_{R}, k_{n}\right) \cdot k_{i}=0,
$$

which vanishes by momentum conservation and transversally of the polarization vectors.

Using standard spinor-helicity notation these soft factors reads (up to normalization constants)

$$
S^{\mathrm{YM}}\left(a, s^{+}, b\right)=\frac{\langle a b\rangle}{\langle a s\rangle\langle s b\rangle}, \quad S^{\mathrm{YM}}\left(a, s^{-}, b\right)=\frac{[a b]}{[a s][s b]} .
$$

\subsection{A more crossing-symmetric KLT relation from soft limits}

A KLT relation with $(n-2) !^{2}$ terms for the $n$-graviton amplitude was recently considered in [2]. This expression has a higher degree of manifest crossing symmetry. Ignoring overall normalization constants, it reads

$$
\begin{aligned}
& M_{n} \approx(-1)^{n} \\
& \frac{\sum_{\sigma, \gamma} \widetilde{A}_{n}(n, \gamma(2, \ldots, n-1), 1) \mathcal{S}[\gamma(2, \ldots, n-1) \mid \sigma(2, \ldots, n-1)]_{p_{1}} A_{n}(1, \sigma(2, \ldots, n-1), n)}{s_{12 \ldots(n-1)}} .
\end{aligned}
$$

On-shell this expression is of course ill-defined since then $s_{12 \ldots n-1}=k_{n}^{2}=0$. However, the numerator also vanishes because of the annihilation property (3.3). In [2] a prescription for taking the on-shell limit such that the formula (4.9) gives the correct $n$-point gravity amplitude was provided. In this section we will demonstrate that the soft limit of gravity amplitudes imply that the numerator and denominator indeed vanish at the same rate. This gives an alternative understanding of why the limit in (4.9) is finite and corresponds to the proper $n$-point gravity amplitude. Note that we are using a $\approx$ to remind ourself that the equality is in terms of a limiting procedure.

We start from the field theory limit of the expression with $(n-3) !^{2}$ terms in $(2.16)$

$$
\begin{aligned}
M_{n}= & (-1)^{n-3} \sum_{\sigma, \gamma} \mathcal{S}[\gamma(2, \ldots, n-2) \mid \sigma(2, \ldots, n-2)]_{k_{1}} \\
& \times A_{n}(1, \sigma(2, \ldots, n-2), n-1, n) \widetilde{A}_{n}(n-1, n, \gamma(2, \ldots, n-2), 1) .
\end{aligned}
$$

Without loss of generality we can assume that leg $n$ has positive helicity. Then, using the soft limit $k_{n}^{+} \rightarrow 0$ for the color-ordered amplitudes in (4.3), we get

$$
\begin{aligned}
\lim _{k_{n}^{+} \rightarrow 0}(-1)^{n-3} M_{n} \approx \sum_{\sigma, \gamma} S^{\mathrm{YM}}(n & \left.-1, n^{+}, \gamma(2)\right) S^{\mathrm{YM}}\left(n-1, n^{+}, 1\right) \mathcal{S}[\gamma(2, \ldots, n-2) \mid \sigma(2, \ldots, n-2)]_{k_{1}} \\
& \times A_{n-1}(1, \sigma(2, \ldots, n-2), n-1) \widetilde{A}_{n-1}(n-1, \gamma(2, \ldots, n-2), 1) .
\end{aligned}
$$


We note that this limit does not affect the momentum kernel. One sees that

$$
\begin{aligned}
S^{\mathrm{YM}}\left(n-1, n^{+}, i\right) S^{\mathrm{YM}}\left(n-1, n^{+}, 1\right) & =\frac{\langle n-1,1\rangle}{\langle n-1, n\rangle\langle n, 1\rangle} \frac{\langle n-1, i\rangle}{\langle n-1, n\rangle\langle n, i\rangle} \\
& =\frac{1}{s_{n, n-1}} \frac{\langle n-1,1\rangle[n, n-1]}{\langle n-1, n\rangle\langle n, 1\rangle} \frac{\langle n-1, i\rangle}{\langle n, i\rangle},
\end{aligned}
$$

and in the denominator $s_{n n-1}=s_{12 \cdots n-2}$. We can thus rewrite the soft limit of the $n$-point gravity amplitude as

$$
\begin{aligned}
& \lim _{k_{n}^{+} \rightarrow 0}(-1)^{n-3} M_{n} \approx \frac{\langle n-1,1\rangle[n, n-1]}{\langle n-1, n\rangle\langle n, 1\rangle} \sum_{i=2}^{n-2} \frac{\langle n-1, i\rangle}{\langle n, i\rangle} \times \\
& \sum_{\sigma, \gamma_{i}} \frac{\widetilde{A}_{n}\left(n-1, i, \gamma_{i}(2, . ., n-2), 1\right) \mathcal{S}\left[i, \gamma_{i}(2, . ., n-2) \mid \sigma(2, . ., n-2)\right]_{k_{1}} A_{n}(1, \sigma(2, . ., n-2), n-1)}{s_{12 \ldots n-2}}
\end{aligned}
$$

where the permutation $\gamma_{i}$ is over the $n-4$ legs $\{2, \ldots, i-1, i+1, \ldots, n-2\}$ with $2 \leq i \leq n-2$. Comparing with the soft limit $k_{n}^{+} \rightarrow 0$ of the graviton amplitude [4, 29]

$\lim _{k_{n}^{+} \rightarrow 0} M_{n}(1,2, \ldots, n-1, n) \approx-\frac{1}{\langle n, 1\rangle\langle n, n-1\rangle} \sum_{i=2}^{n-2} \frac{\langle n-1, i\rangle}{\langle n, i\rangle}\langle 1, i\rangle[i, n] M_{n-1}(1,2, \ldots, n-1)$,

we deduce that the terms at the right-hand-side must satisfy

$$
\begin{array}{r}
\sum_{\sigma, \gamma_{i}} \frac{\widetilde{A}_{n}\left(n-1, i, \gamma_{i}(2, . ., n-2), 1\right) \mathcal{S}\left[i, \gamma_{i}(2, . ., n-2) \mid \sigma(2, . ., n-2)\right]_{k_{1}} A_{n}(1, \sigma(2, . ., n-2), n-1)}{s_{12 \ldots n-2}} \\
\approx-\frac{\langle 1 i\rangle[i n]}{\langle 1, n-1\rangle[n-1, n]} M_{n-1}, \quad(4.15)
\end{array}
$$

Summing over $i$ and using the momentum conservation identity $\sum_{i=2}^{n-1}\langle 1 i\rangle[i n]=0$ one gets the expression (4.9) of [2] (omitting the overall sign factor)

$$
\begin{aligned}
& M_{n-1} \\
& \approx \sum_{\sigma, \gamma} \frac{\widetilde{A}_{n}(n-1, \gamma(2, \ldots, n-2), 1) \mathcal{S}[\gamma(2, \ldots, n-2) \mid \sigma(2, \ldots, n-2)]_{k_{1}} A_{n}(1, \sigma(2, \ldots, n-2), n-1)}{s_{12 \ldots n-2}} .
\end{aligned}
$$

Using that

$$
\frac{\langle 1 i\rangle[i n]}{\langle 1, n-1\rangle[n-1, n]}=\frac{s_{i q}}{s_{n-1, q}}, \quad \text { with } \quad q \equiv|1\rangle[n \mid
$$

it follows from the calculations in [25], that the above soft limit procedure gives an equivalent description of eq. (4.16) as the off-shell regularization introduced in [2] would. Especially, note that the auxiliary momentum $q$ satisfy all the requirements $[2,25]$ for an off-shell regularization, i.e. $q^{2}=k_{1} \cdot q=0$ and $q \cdot k_{n-1} \neq 0$. 


\section{Color and its kinematic factors}

The usual color decomposition of tree-level amplitudes in gauge theory is given by

$$
A_{n}^{\mathrm{YM}}=\sum_{\sigma} \operatorname{tr}\left[T^{1} T^{\sigma(2)} \cdots T^{\sigma(n)}\right] A_{n}(1, \sigma(2), \ldots, \sigma(n)),
$$

where $A_{n}$ are the color-ordered vector amplitudes and $T^{i}$ generators of the gauge group. The cyclic and the reverse properties of the trace imply that there are $(n-1) ! / 2$ independent color factors.

An alternative form of the tree-level gauge amplitude is [31]

$$
A_{n}^{\mathrm{YM}}=\sum_{\delta} c_{1|\delta(2, \ldots, n-1)| n} A_{n}(1, \delta(2, \ldots, n-1), n),
$$

where the coefficients are products of the structure constants $\left(f^{a}\right)_{i j}=\operatorname{tr}\left(\left[T^{i}, T^{j}\right] T^{a}\right)$

$$
c_{\beta_{1}\left|\alpha_{1} \cdots \alpha_{n-1}\right| \beta_{n}}=f_{\beta_{1} i_{1}}^{\alpha_{1}} f_{i_{1} i_{3}}^{\alpha_{2}} \cdots f_{j_{n-1} \beta_{n}}^{\alpha_{n-1}} .
$$

An extension of this representation to one-loop order was provided in [32]. The equivalence between the original form (5.1) and (5.2) relies on the Kleiss-Kuijf relations satisfied by color-ordered amplitudes [33], e.g.

$$
A_{n}(1,2,3 \ldots, n)+A_{n}(1,3,4, \ldots, n, 2)+\cdots+A_{n}(1, n, 2,3, \ldots, n-1)=0 .
$$

Because of the annihilation property in (3.3), the color coefficients in (5.2) can be arbitrarily shifted as

$$
c_{1|\delta(2, \ldots, n-1)| n} \rightarrow c_{1|\delta(2, \ldots, n-1)| n}+\sum_{\sigma} g_{\sigma} \times \mathcal{S}[\delta(2, \ldots, n-1) \mid \sigma(2, \ldots, n-1)]_{k_{n}} .
$$

The coefficients $g_{\sigma}$ can be constants or (not even necessarily gauge invariant) functions of the external polarizations and momenta. Among the $(n-2) ! g_{\sigma}$-coefficients $(n-3) !$ are redundant, because they correspond to shifts in the kernel of the annihilation property, and only $(n-3) \times(n-3)$ ! coefficients are therefore necessary in (5.5). The form of the color factors in (5.3) is a specific choice of representation. We will illustrate this point at the end of this section.

Thanks to the duality between color and kinematic factors, as noted by Bern-CarrascoJohansson [5], one expects that the gravity tree-level amplitudes take a similar form [18, 34]

$$
M_{n}=(-1)^{n-3} \sum_{\sigma} n_{1|\sigma(2) \cdots \sigma(n-1)| n} A_{n}(1, \sigma(2, \ldots, n-1), n) .
$$

To derive such a representation for the gravity amplitude we will define $\left(i_{2}^{0}, \ldots, i_{n-1}^{0}\right)=$ $(2,3,4, \ldots, n-1)$ to be the identity, $\left(i_{2}^{1}, \ldots, i_{n-1}^{1}\right)=(n-1,2,3, \ldots, n-2)$ the cyclic right permutation of order $n-1$, and $\left(i_{2}^{k}, \ldots, i_{n-1}^{k}\right)=(n-k, n-k+1 \cdots, n-1-k)$ the $k$ th iteration of the cyclic right permutation (the notation is to be understood modulo $n-2$ as $\left.i_{n-1}^{2}=2\right)$. 
We start by considering the symmetrized version of the $n$-graviton amplitude

$$
M_{n}(1,2, \ldots, n)=\frac{1}{n-2} \sum_{k=0}^{n-3} M_{n}\left(1, i_{2}^{k}, \ldots, i_{n-1}^{k}, n\right) .
$$

One can consider a more general averaging with non-equal weight factors. Plugging in the $(n-3) !^{2}$ formula $(2.16)$ for the $n$-point graviton amplitude in this expression we get

$$
\begin{aligned}
M_{n}= & \frac{(-1)^{n-3}}{n-2} \sum_{j=0}^{n-3} \sum_{\sigma, \gamma} \mathcal{S}\left[\gamma\left(i_{2}^{j}, \ldots, i_{n-2}^{j}\right) \mid \sigma\left(i_{2}^{j}, \ldots, i_{n-2}^{j}\right)\right]_{k_{1}} \\
& \times A_{n}\left(1, \sigma\left(i_{2}^{j}, \ldots, i_{n-2}^{j}\right), i_{n-1}^{j}, n\right) \widetilde{A}_{n}\left(1, \gamma\left(i_{2}^{j}, \ldots, i_{n-2}^{j}\right), n, i_{n-1}^{j}\right) .
\end{aligned}
$$

The same manipulations can be done starting from the $j$-dependent formula in (2.15). The equivalence of the resulting expressions is obtained by a repeated use of the monodromy relations $[8,15]$ between the color-ordered amplitudes. Defining the coefficients

$$
\begin{aligned}
& n_{1\left|\sigma\left(i_{2}^{j}, \ldots, i_{n-2}^{j}\right) i_{n-1}^{j}\right| n} \\
& \equiv \frac{1}{n-2} \sum_{\gamma} \mathcal{S}\left[\gamma\left(i_{2}^{j}, \ldots, i_{n-2}^{j}\right) \mid \sigma\left(i_{2}^{j}, \ldots, i_{n-2}^{j}\right)\right]_{k_{1}} \times \widetilde{A}_{n}\left(1, \gamma\left(i_{2}^{j}, \ldots, i_{n-2}^{j}\right), n, i_{n-1}^{j}\right),
\end{aligned}
$$

and remarking that the sum over all $\sigma$-permutations composed with the cyclic permutations $i^{k}$ provide all permutations of $\{2, \ldots, n-1\}$, we can rewrite (5.8) as

$$
M_{n}=(-1)^{n-3} \sum_{\delta} n_{1|\delta(2, \ldots, n-1)| n} A_{n}(1, \delta(2, \ldots, n-1), n),
$$

with

$$
\begin{aligned}
n_{1|\delta(2, \ldots, n-1)| n}= & \frac{1}{n-2} \sum_{\gamma} \mathcal{S}[\gamma(\delta(2), \ldots, \delta(n-2)) \mid \delta(2), \ldots, \delta(n-2)]_{k_{1}} \times \\
& \times \widetilde{A}_{n}(1, \gamma(\delta(2), \ldots, \delta(n-2)), n, \delta(n-1)) .
\end{aligned}
$$

This provides the form of the $n$-point graviton amplitude similar to (5.2), where the color factors have been replaced by kinematic factors.

We remark that the manipulations leading to (5.10) and (5.11) are valid in string theory as well, with the replacement of the field theory $\mathcal{S}$ by its stringy version $\mathcal{S}_{\alpha^{\prime}}$ and the field theory amplitudes $A_{n}$ and $\widetilde{A}_{n}$ by the correspond string amplitudes.

There are $(n-2)$ ! color and kinematic coefficients in the decomposition in (5.2) and (5.6) which is the same as the number of independent numerator factors in the BCJ decomposition of amplitudes in terms of cubic vertices [5, 18, 34]. The expression (5.11) provides a possible constructive definition for these independent numerator factors.

We illustrate the construction of the color coefficient in (5.2) and the kinematic coefficients (5.6) in the case of the four points. By applying (5.11) we have

$$
\hat{c}_{1|23| 4}=\frac{1}{2} \mathcal{S}[2 \mid 2]_{k_{1}} A_{4}^{s}(1243), \quad \hat{c}_{1|32| 4}=\frac{1}{2} \mathcal{S}[3 \mid 3]_{k_{1}} A_{4}^{s}(1342),
$$


where the scalar amplitudes are defined by [5]

$$
A_{4}^{s}(1243)=A_{4}^{s}(1342)=\frac{c_{s}}{s}+\frac{c_{u}}{u},
$$

with the numerator factors given by $c_{s}=-\sum_{i} f_{12}^{i} f_{34}^{i}$ and $c_{u}=\sum_{i} f_{31}^{i} f_{24}^{i}$. Therefore we get

$$
\hat{c}_{1|23| 4}=\frac{1}{2}\left(c_{s}+\frac{s}{u} c_{u}\right), \quad \hat{c}_{1|32| 4}=\frac{1}{2}\left(c_{u}+\frac{u}{s} c_{s}\right) .
$$

These coefficients have a non-local momentum dependent form which can be removed by using the freedom in (5.5). We can, for instance, shift these coefficients, without modifying the amplitude, by

$$
c_{1|\sigma(23)| 4}=\hat{c}_{1|\sigma(23)| 4}+\frac{1}{2}\left(\frac{c_{u}}{s u}-\frac{c_{s}}{s^{2}}\right) \mathcal{S}[\sigma(2,3) \mid 3,2]_{k_{4}},
$$

to obtain the form of the coefficients in (5.3)

$$
c_{1|23| 4}=c_{s}, \quad c_{1|32| 4}=c_{u} .
$$

The same manipulations can be done for the kinematic coefficient when applying (5.11) with the vector amplitudes

$$
A_{4}(1243)=\frac{n_{s}}{s}+\frac{n_{u}}{u}, \quad A_{4}(1234)=-\frac{n_{s}}{s}+\frac{n_{t}}{t}, \quad A_{4}(1324)=-\frac{n_{u}}{u}-\frac{n_{t}}{t} .
$$

Exchanging the vector and the scalar amplitudes in these formulaes exchange the color and kinematic coefficients.

\section{Conclusions}

This paper has been devoted to the properties of the momentum kernel that lies behind closed-open string factorization. This kernel is the central object in the relation between gravity and gauge theory amplitudes in both string theory and field theory. We have shown that this kernel defines a set of linear equations that annihilate color-ordered Yang-Mills amplitudes: it is the generator of monodromy relations between these amplitudes. The kernel has also an interesting connection relating to kinematic factors.

Although no natural origin of this momentum kernel is known from the structure of the (effective) gravity and gauge theory Lagrangians, we have shown that this object arises naturally when one constructs closed (type II or heterotic) string amplitudes. This parallels (and complements) the $S$-matrix based motivation for this kernel in [2]. The very simple product form of this kernel makes it easy to implement the KLT-form of the amplitudes in (2.15), the annihilation relations in (3.3), and the definition of the kinematic factors in (5.11).

Our string-based derivation of the annihilation property and, consequently, the monodromy relations between color-ordered amplitudes did not use any detailed properties of the spectrum of the theory. The momentum kernel follows in a universal way from the phases of the operator product expansion. We therefore expect that such a kernel will enter in relations between ordered correlators in other contexts that are based on a conformal field theory, as in solid state physics. It would be interesting to have this possible application elucidated in greater detail. 


\section{Acknowledgments}

P.V. would like to thank the Niels Bohr International Academy, where part of this work was carried out, for hospitality. This research has been supported in part by the French Embassy of Denmark.

Open Access. This article is distributed under the terms of the Creative Commons Attribution Noncommercial License which permits any noncommercial use, distribution, and reproduction in any medium, provided the original author(s) and source are credited.

\section{References}

[1] H. Kawai, D.C. Lewellen and S.H. Henry Tye, A relation between tree amplitudes of closed and open strings, Nucl. Phys. B 269 (1986) 1 [SPIRES].

[2] N.E.J. Bjerrum-Bohr, P.H. Damgaard, B. Feng and T. Sondergaard, Gravity and Yang-Mills amplitude relations, Phys. Rev. D 82 (2010) 107702 [arXiv: 1005.4367] [SPIRES].

[3] N.E.J. Bjerrum-Bohr, P.H. Damgaard, B. Feng and T. Sondergaard, Proof of gravity and Yang-Mills amplitude relations, JHEP 09 (2010) 067 [arXiv: 1007.3111] [SPIRES].

[4] Z. Bern, L.J. Dixon, M. Perelstein and J.S. Rozowsky, Multi-leg one-loop gravity amplitudes from gauge theory, Nucl. Phys. B 546 (1999) 423 [hep-th/9811140] [SPIRES].

[5] Z. Bern, J.J.M. Carrasco and H. Johansson, New relations for gauge-theory amplitudes, Phys. Rev. D 78 (2008) 085011 [arXiv:0805.3993] [SPIRES].

[6] T. Sondergaard, New relations for gauge-theory amplitudes with matter, Nucl. Phys. B 821 (2009) 417 [arXiv:0903.5453] [SPIRES].

[7] Y. Jia, R. Huang and C.-Y. Liu, U(1)-decoupling, KK and BCJ relations in $\mathcal{N}=4 S Y M$, Phys. Rev. D 82 (2010) 065001 [arXiv:1005.1821] [SPIRES].

[8] N.E.J. Bjerrum-Bohr, P.H. Damgaard and P. Vanhove, Minimal basis for gauge theory amplitudes, Phys. Rev. Lett. 103 (2009) 161602 [arXiv:0907.1425] [SPIRES].

[9] S. Stieberger, Open $\&$ closed vs. pure open string disk amplitudes, arXiv:0907.2211 [SPIRES].

[10] E. Plahte, Symmetry properties of dual tree-graph $N$-point amplitudes, Nuovo Cim. A 66 (1970) 713 [SPIRES].

[11] B. Feng, R. Huang and Y. Jia, Gauge amplitude identities by on-shell recursion relation in S-matrix program, arXiv: 1004.3417 [SPIRES].

[12] Z. Bern, A. De Freitas and H.L. Wong, On the coupling of gravitons to matter, Phys. Rev. Lett. 84 (2000) 3531 [hep-th/9912033] [SPIRES].

[13] C.R. Mafra, Simplifying the tree-level superstring massless five-point amplitude, JHEP 01 (2010) 007 [arXiv:0909.5206] [SPIRES].

[14] S.H. Henry Tye and Y. Zhang, Dual identities inside the gluon and the graviton scattering amplitudes, JHEP 06 (2010) 071 [arXiv: 1003.1732] [SPIRES].

[15] N.E.J. Bjerrum-Bohr, P.H. Damgaard, T. Sondergaard and P. Vanhove, Monodromy and Jacobi-like relations for color-ordered amplitudes, JHEP 06 (2010) 003 [arXiv:1003.2403] [SPIRES]. 
[16] C. Cheung, D. O'Connell and B. Wecht, BCFW recursion relations and string theory, JHEP 09 (2010) 052 [arXiv: 1002.4674] [SPIRES].

[17] R.H. Boels, D. Marmiroli and N.A. Obers, On-shell recursion in string theory, JHEP 10 (2010) 034 [arXiv: 1002 .5029] [SPIRES].

[18] Z. Bern, T. Dennen, Y.-T. Huang and M. Kiermaier, Gravity as the square of gauge theory, Phys. Rev. D 82 (2010) 065003 [arXiv: 1004. 0693] [SPIRES].

[19] D. Vaman and Y.-P. Yao, Constraints and generalized gauge transformations on tree-level gluon and graviton amplitudes, JHEP 11 (2010) 028 [arXiv:1007.3475] [SPIRES].

[20] Z. Bern, J.J.M. Carrasco and H. Johansson, Perturbative quantum gravity as a double copy of gauge theory, Phys. Rev. Lett. 105 (2010) 061602 [arXiv:1004.0476] [SPIRES].

[21] P. Vanhove, The critical ultraviolet behaviour of $\mathcal{N}=8$ supergravity amplitudes, arXiv: 1004.1392 [SPIRES].

[22] N.E.J. Bjerrum-Bohr, P.H. Damgaard, B. Feng and T. Sondergaard, New identities among gauge theory amplitudes, Phys. Lett. B 691 (2010) 268 [arXiv:1006.3214] [SPIRES].

[23] S.H. Tye and Y. Zhang, Comment on the identities of the gluon tree amplitudes, arXiv: 1007.0597 [SPIRES].

[24] B. Feng and S. He, KLT and new relations for $\mathcal{N}=8$ SUGRA and $\mathcal{N}=4 S Y M$, JHEP 09 (2010) 043 [arXiv: 1007.0055] [SPIRES].

[25] B. Feng, S. He, R. Huang and Y. Jia, Note on new KLT relations, JHEP 10 (2010) 109 [arXiv:1008.1626] [SPIRES].

[26] H. Elvang and M. Kiermaier, Stringy KLT relations, global symmetries and $E_{7(7)}$ violation, JHEP 10 (2010) 108 [arXiv: 1007.4813] [SPIRES].

[27] V.S. Dotsenko and V.A. Fateev, Conformal algebra and multipoint correlation functions in $2 D$ statistical models, Nucl. Phys. B 240 (1984) 312 [SPIRES].

[28] V.S. Dotsenko and V.A. Fateev, Four point correlation functions and the operator algebra in the two-dimensional conformal invariant theories with the central charge $C<1$, Nucl. Phys. B 251 (1985) 691 [SPIRES].

[29] S. Weinberg, Infrared photons and gravitons, Phys. Rev. 140 (1965) B516 [SPIRES].

[30] F.A. Berends and W.T. Giele, Multiple soft gluon radiation in parton processes, Nucl. Phys. B 313 (1989) 595 [SPIRES].

[31] V. Del Duca, A. Frizzo and F. Maltoni, Factorization of tree QCD amplitudes in the high-energy limit and in the collinear limit, Nucl. Phys. B 568 (2000) 211 [hep-ph/9909464] [SPIRES].

[32] V. Del Duca, L.J. Dixon and F. Maltoni, New color decompositions for gauge amplitudes at tree and loop level, Nucl. Phys. B 571 (2000) 51 [hep-ph/9910563] [SPIRES].

[33] R. Kleiss and H. Kuijf, Multi-gluon cross-sections and five jet production at hadron colliders, Nucl. Phys. B 312 (1989) 616 [SPIRES].

[34] M. Kiermaier, Gravity as the square of gauge theory, talk given at Amplitudes 2010, Queen Mary University, London U.K. May 4-7 2010. 